\section{NETs: Kombination in Phase-III-Studie erfolgreich}

Zwei Arzneien hatten in jüngerer Zeit Erfolge gegenüber neuroendokrinen Tumoren (NETs) vorzuweisen. Eine Kombination von Everolimus und Octreotid-LAR hat nun auch in einer Phase-III-Studie ansehnliche Ergebnisse bei Karzinoiden verschiedenen Ursprungs erzielt.

$\mathrm{D}$ er orale mTOR-Inhibitor Everolimus hatte Patienten mit fortgeschrittenen NETs des Pankreas zu einem um 6,4 Monate längeren progressionsfreien Überleben verholfen. Eine internationale Arbeitsgruppe unter deutscher Beteiligung hat Everolimus deshalb in einer PhaseIII-Studie in Kombination mit OctreotidLAR bei NETs getestet, die mit einem Karzinoid-Syndrom assoziiert waren.

In der Doppelblindstudie erhielten die Patienten im Verum-Arm täglich $10 \mathrm{mg}$ Everolimus und alle 28 Tage $30 \mathrm{mg}$ Octreotid LAR i.m., im Kontroll-Arm statt
Everolimus ein Placebo. Die Patienten hatten NETs von niedrigem oder intermediärem Grad in verschiedenen Organen - mehr als die Hälfte im Dünndarm, weitere $15 \%$ in der Lunge. Primärer Endpunkt war das progressionsfreie Überleben.

Von 429 randomisierten Patienten brachen 357 im Laufe der Studie die Behandlung ab. Das mediane progressionsfreie Überleben lag bei 16,4 Monaten im Everolimus- und bei 11,3 Monaten im Placebo-Arm (Hazard Ratio [HR] 0,77). Besonders stark profitierten Patienten mit primären Kolontumoren (HR 0,39). Die
Zugabe von Everolimus zur OctreotidTherapie ging mit relativ milden Symptomen vom Grad 1 oder 2 einher - gehäuft traten v.a. Stomatitis (62 vs. $14 \%$ ), Hautausschläge ( 37 vs. $12 \%$ ), Fatigue (31 vs. $23 \%$ ) sowie Diarrhö (27 vs. $16 \%$ ) auf.

Fazit: Everolimus, zusätzlich zu Octreotid-LAR gegeben, konnte das progressionsfreie Überleben von Patienten mit fortgeschrittenen NETs verschiedener Ausgangsgewebe verbessern. Der Effekt war besonders stark bei Patienten mit primären Kolontumoren. Christina Berndt

Pavel ME et al. Everolimus plus octreotide long-acting repeatable for the treatment of advanced neuroendocrine tumours associated with carcinoid syndrome (RADIANT-2): a randomised, placebo-controlled, phase 3 study. Lancet. 2011;378(9808):2005-12.

\title{
p53-Mutation auch bei GIST relevant
}

Die Bedeutung von KIT- und PDGFRA-Mutationen bei gastrointestinalen Stromatumoren (GIST) ist bekannt (Abb.). Jetzt ging eine Metaanalyse der möglichen Bedeutung von p53-Mutationen bei GIST nach.

C hinesische Onkologen analysierten alle publizierten Daten zur p53-Expression im Zusammenhang mit der Malignität bei GIST. In neun Studien mit 768

Patienten erfolgte die Analyse nach Malignität gemäß den von den National Institutes of Health (NIH) definierten vier Risikogruppen: sehr geringes (very low,

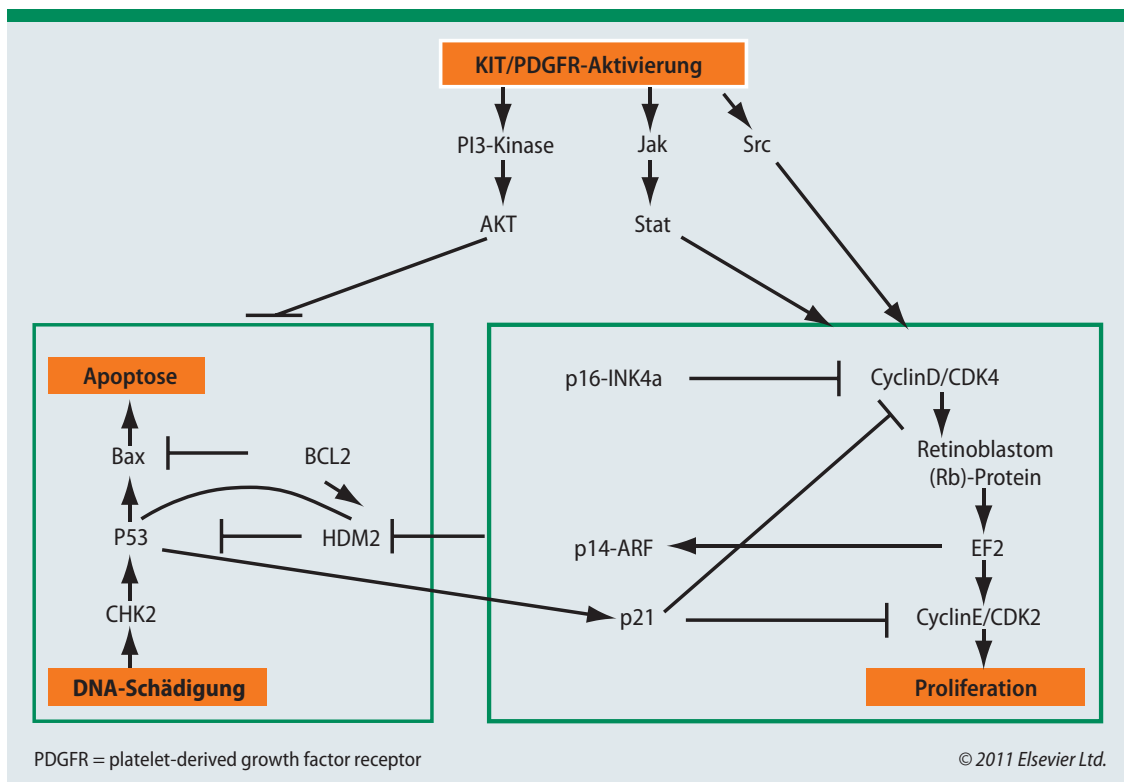

Abb.: Die Mutation von KIT/PDGFR fördert die Proliferation und hemmt die Apoptose der Zelle über verschiedene Signalwege.
NIH-VL), geringes (low, NIH-L), mittleres (intermediate, NIH-I) und hohes $\mathrm{Ri}$ siko (high, NIH-H). Eine p53-Überexpression in der Gruppe mit sehr geringem Risiko war nicht signifikant häufiger als in der Gruppe mit niedrigem Risiko (Odds ratio [OR] 0,38; 95\%-Konfidenzintervall [95\%-KI] 0,11-1,28; $\mathrm{p}=0,12$ ). Das galt auch für die NIH-I-Gruppe im Vergleich zur NIH-H-Gruppe, wenn sich hier auch schon ein Trend abzuzeichnen schien (OR 0,62; 95\%-KI 0,37-1,02; p = $0,06)$. Am deutlichsten wurde der mögliche Zusammenhang beim Vergleich von NIH-L- mit NIH-I-GIST (OR 0,44; 95\%KI 0,24-0,82; $\mathrm{p}=0,009$ ).

Fazit: Insgesamt bleiben die Autoren vorsichtig: Die teils kleinen Patientenzahlen, unterschiedliche Antikörper und die Heterogenität der Studien lassen keine definitiven Rückschlüsse auf die Rolle der p53-Überexpression für die Malignitiät der GIST zu. Die Ergebnisse der Metaanalyse sprechen aber für eine Korrelation von p53-Überexpression und Malignitätsrisiko, am deutlichsten bei GIST mit intermediärem Risiko. Friederike Klein

Zong L et al. Correlation between p53 expression and malignant risk of gastrointestinal stromal tumors: evidence from 9 studies. Eur J Surg Oncol. 2012;38(3):189-95. 